\title{
The USE OF CePHalosporins as ProphylaCtiC Antibiotic ToWARdS The Prevention of Surgical WOUND INFECTIONS
}

\author{
Pushkar Moyal Singh ${ }^{1}$, Joh-Clinton Thomas ${ }^{2}$ and Abhilash Thomas ${ }^{2 *}$ \\ ${ }^{1}$ Department of Pharmacology, Arya College of Pharmacy, Jaipur-302028, Rajasthan, India \\ ${ }^{2}$ Department of Pharmacy Practice, N.E. T. Pharmacy College, Raichur-584103, Karnataka, India \\ *E-mail: abhi0882@gmail.com \\ Tel.: +91 7402100767.
}

Received: Mar 14, 2017 / Revised: Apr 15, 2017 / Accepted: Apr 16, 2017

\begin{abstract}
Antimicrobial prophylaxis is indicated for procedures to reduce the incidence of postoperative wound infections but additional antibiotic use increases the emergence of antimicrobial resistance. The present study assess the current use of cephalosporin as prophylactic antibiotic either single agent or combination with other antimicrobials in patients undergoing surgical procedures in a tertiary care hospital. A total of 1500 surgeries were studied including elective and non elective cases. The data collection was done from the source documents such as patient case report, anesthesia chart, preoperative checklist, medication chart and surgical safety checklist. The patient demographics such as age, body weight, sex, name of the surgical procedure, date of surgery, type of surgery, pre operative antibiotic, time of administration etc. were collected. The second generation Cephalosporins was used mostly for prophylaxis (n-820) followed by third generation Cephalosporins (n-421). Even though, the number of clean surgeries was high $(91 \%)$ the post operative antibiotic dose was not discontinued $\geq 24 \mathrm{~h}(68 \%)$. Cefazolin, the CDC recommended prophylactic agent was used only $4 \%$ patients. However, in the present study single Cefoperazone with Sulbactam (17\%) and with a combination of aminoglycoside, Ofloxacin and Metronidazole with Cefoperazone was the most common regimen used. The results highlight the challenges of disseminating evidence based protocols systematically into routine clinical practice. We suggest various measures to improve appropriateness of prescriptions and adherence include development of evidence based guidelines in collaboration with surgeons, increased outcome based research to document benefits of appropriate antibiotic use.
\end{abstract}

Key words: Prophylaxis, cephalosporins, aminoglycosides, sulbactam, adherence.

\section{INTRODUCTION}

Prophylaxis has become the standard of care for contaminated and clean contaminated surgery and for surgery involving insertion of artificial devices. Appropriate antibiotic prophylaxis can reduce the risk of postoperative wound infections, but additional antibiotic use also increases the selective pressure favoring the emergence of antimicrobial resistance. Surgical site infections (SSIs) account for approximately
$15 \%$ of nosocomial infections and are associated with prolonged hospital stays and increased costs especially with resistant pathogens, such as Methicillin-resistant Staphylococcus aureus (MRSA) and Candida species (Berard and Gandon, 1964). Factors influencing the development of SSI's include bacterial inoculums and virulence, host defenses, preoperative care, and intra operative management (Horan and Culver, 1993; Olson and Lee, 1990). 
Principles of prophylaxis include providing effective levels of antibiotics in the decisive interval, and, in most instances, limiting the course to intra operative coverage only. This prophylaxis should be precise to determine the bacterial flora most likely to cause postoperative infection to choose an antibiotic, based on the steps above, with the narrowest antibacterial spectrum required, to choose the less expensive drug if two drugs are otherwise of equal antibacterial spectrum, efficacy, toxicity, and ease of administration, to administer dose at the right time, to administer antibiotics for a short period (one dose if surgery of four hours duration or less) to avoid antibiotics likely to be of use in the treatment of serious sepsis (Page et al 1993), to review antibiotic prophylaxis protocols regularly as both cost and hospital antibiotic resistance patterns may change and to decide if prophylaxis is appropriate (Bratzler et al 2005). The goals of prophylactic administration of antibiotics to surgical patients are to reduce the incidence of surgical site infection, to use antibiotics in a manner that is supported by evidence of effectiveness, to minimize the effect of antibiotics on the patient's normal bacterial flora.

To minimize adverse effects, to cause minimal change to the patient's host defenses, to aseptic technique is adhered to throughout the surgical procedure. It is also recommended that surgeon preferences for the type of antibiotics to be administered is based on the site of the surgical procedure, potential risk factors and based on the hospital microbial flora (Kulkarni and Kochhar, 2005). This study is to assess the current use of cephalosporin as prophylactic antibiotic either single agent or combination with other antimicrobials among patients undergoing surgical procedures in a tertiary care hospital.

Also, this study aims to measure the compliance with quality indicator (current policy) in the delivery of surgical prophylaxis and to assess the impact of a change to the hospital's surgical prophylaxis policy.

\section{MATERIALS ANS METHODS}

\section{Study design and site}

A prospective observational study, conducted in a tertiary care, India.

\section{Study period}

The study was conducted over a period of 9 months.

\section{Study population}

Subjects of age 18-60 yr undergoing elective as well as emergency surgery.

\section{Study protocol}

The observational study includes all patients of any age who undergoes surgery, both elective and non elective or emergency surgery. The data collection is conducting prospectively from the department of cardiology, thoracic surgery, minimal accessible surgery and bariatric surgery, gynecology, neurology; urology includes renal transplant, ophthalmology, plastic and cosmetic surgery and orthopedic surgery. Patients with current infections on single or multiple antibiotic therapies are also included (Figure 1, 2).

\section{Source of data}

Data source includes patient case report, anesthesia chart, preoperative checklist, medication chart and surgical safety checklist. The data particulars collected include:

- Patient demographics such as age, body weight, sex

- Department of surgery

- Name of the surgical procedure to be done

- Date of surgery

- Type of surgery whether it is clean, clean contaminated, contaminated or dirty

- Name of pre operative antibiotic, if any

- Name of perioperative antibiotic administered

- Time of administration

- Time of surgery

The follow up has been done on:

- Additionally administered doses in between the surgery in case of surgery prolonged $>4$ h.

- Post operative antibiotic given- Name of antibiotic and time of administration,

- Post operative antibiotic prophylaxis duration,

- Whether the antibiotic given as instructed by the policy or not,

- Compliances as per the current hospital policy 


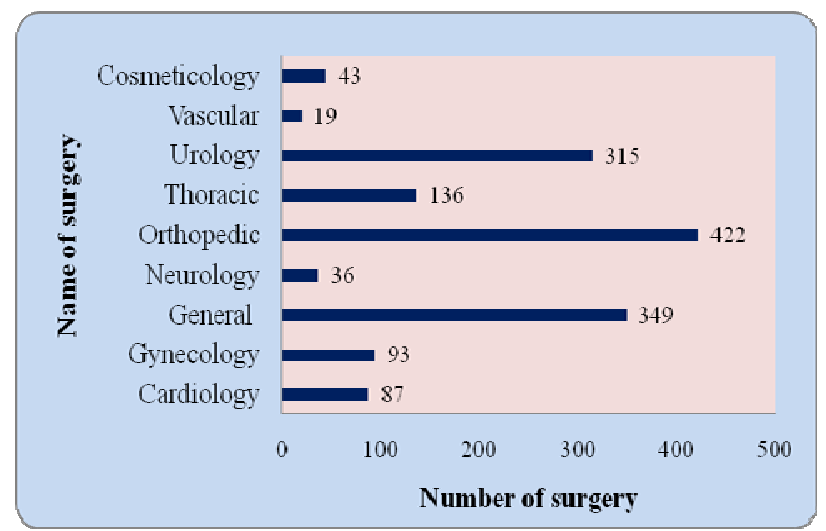

Fig. 1. Number of surgeries from various depts (values expressed as number)

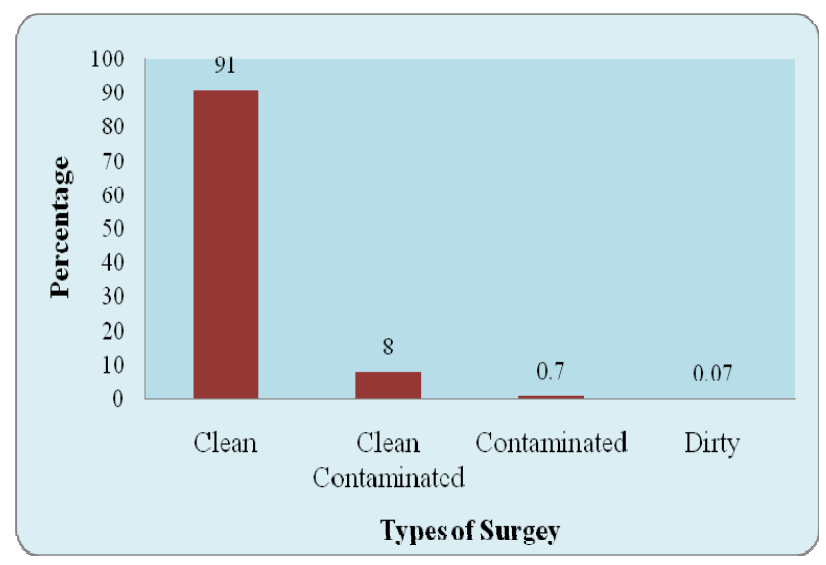

Fig. 2. Types of surgeries (values expressed as percentage)

\section{Analysis and interpretation}

The collected data was analyzed and the values were interpreted as number and percentages.

\section{RESULTS AND DISCUSSION}

In this study, $76 \%$ of surgeries had used Cephalosporins for prophylaxis as single agent or with combination to prevent the surgical site infection, across all surgery groups' viz. clean, contaminated and dirty surgeries (Table 1).

The use of preoperative Cephalosporin as antibiotic prophylaxis is well established. Despite this, study has shown that optimal practice is not achieved in hospitals. The majority $(83 \%)$ of patients received antibiotic prophylaxis prior to surgery in which $76 \%$ cephalosporin used of total antibiotics prophylaxis in which Cefazolin (first-generation cephalosporin), Cefuroxime, (2nd generation Cephalosporin's) have been widely recommended with success. Third generation Cephalosporin's, Cefotaxime, Cefoperazone, Ceftriaxone and Ceftazidime which are generally not recommended for surgical prophylaxis also have been used (Bratzler et al 2005). Despite of all recommendations, the results of our survey showed that Cefazolin, Cefoperazone and Cefuroxime were commonly preferred for surgical prophylaxis (Figure 3).

Table1. Specialty wise distribution types of surgeries

\begin{tabular}{|c|c|c|c|c|c|}
\hline \multicolumn{2}{|c|}{ Department } & \multicolumn{4}{|c|}{ Types of Surgery } \\
\hline S. No. & Specialty & Clean & $\begin{array}{c}\text { Clean } \\
\text { contaminated }\end{array}$ & Contaminated & Dirty \\
\hline 1 & Cardiology (n-87) & 85 & 1 & 1 & 0 \\
\hline 2 & Gynecology (n-93) & 80 & 13 & 0 & 0 \\
\hline 3 & General Surgery (n-349) & 307 & 37 & 4 & 1 \\
\hline 4 & Neurology (n-36) & 35 & 1 & 0 & 0 \\
\hline 5 & Orthopedics (n-422) & 408 & 12 & 2 & 0 \\
\hline 6 & Thoracic (n-136) & 115 & 20 & 1 & 0 \\
\hline 7 & Urologic (n-315) & 288 & 26 & 1 & 0 \\
\hline 8 & Vascular (n-19) & 13 & 5 & 1 & 0 \\
\hline 9 & Cosmeticology (n-43) & 40 & 3 & 0 & 0 \\
\hline \multicolumn{7}{r}{} & Total & $1371(91 \%)$ & $118(8 \%)$ & $10(0.7 \%)$ & $1(0.07 \%)$ \\
\hline
\end{tabular}

Cefazolin, the CDC recommended prophylactic agent was used only $4 \%$ patients. However, in the present study single Cefoperazone with Sulbactam (17\%) and with a combination of aminoglycoside, Ofloxacin and Metronidazole with Cefoperazone was the most common regimen used, while the use of third generation Cephalosporin's alone were also common. In case of combinations of two antimicrobial agents, Cephalosporin's in combination with an anti-anaerobic agent was the preferred (Manian and Meyer, 1990) two drug combination for all types of surgeries followed by Cephalosporin's in combination with Amino glycosides and it is also used as combination with Quinolones. In case of combinations of three antimicrobial 


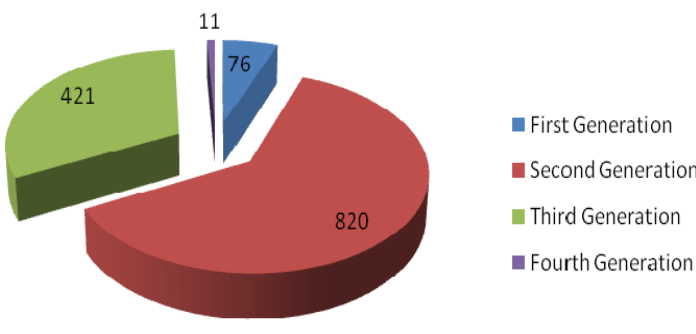

Fig. 3. Generation of Cephalosporins used in surgeries

agents, Cephalosporin's in combination with an amino glycoside and an anti-anaerobic agent was the preferred three drug combination in all types of surgeries (McDonald et al 1998; ASHP, 1999). In this study mostly single cephalosporin antibiotics used as prophylaxis but in few cases combination with Amikacin and Metronidazole also used to cover gram positive, gram negative and anaerobic bacteria surgical site infection (Table 2).

Table 2. Distribution of the antibiotics used

\begin{tabular}{|c|c|}
\hline Cephalosporin & $1328(76 \%)^{*}$ \\
\hline Other & $419(24 \%)$ \\
\hline Single & $1547(89 \%)$ \\
\hline Combination & $200(11 \%)$ \\
\hline
\end{tabular}

*Values expressed as number (\%)
Among the study participants $11 \%$ received antimicrobial combinations in all the surgical departments and $2 \%$ patients received three or more drugs together. Although the use of two or more antimicrobials in combination may have a certain rationale (Platt et al 1990), potentially harmful aspects of such inappropriate antibiotic combinations include the emergence of resistant bacteria, super-infection, the risks of toxic and allergic reaction and increased cost of therapy; further all patients undergoing hernia repair received preoperative antibiotics while no such prophylaxis is recommended. Third generation cephalosporin's were prescribed to almost third half of all the patients who received preoperative antibiotics (Figure 3), which was inappropriate. According to the guidelines, Cefazolin and Cefuroxime should be used for prophylaxis but in this study showed that only in $60 \%$ procedure follow this and $32 \%$ of study participants received Cefotaxime, Cefoperazone, Ceftazidime and Ceftriaxone prior to their surgery. To reduce the emergence of resistance and also because broad spectrum antibiotics may be required later if patient develops serious sepsis (De Lalla, 2006) third generation are to be avoided in surgical prophylaxis (Akalin, 2002). The combination of Cefazolin and Amikacin was used most commonly in this study. Gram-negative bacteria involved in these surgeries are mostly enteric Gram-negative bacilli and Cefazolin alone can cover pathogens; thus there is no need for combination (Hollenbeak et al 2002) (Table 3).

Table 3. Procedures and microorganisms isolated from wounds

\begin{tabular}{|c|c|c|}
\hline S. No. & Name of Surgery/Procedure/Diagnosis & Microorganisms \\
\hline 1 & $\begin{array}{c}\text { Degenerative Disc disease } \\
\text { (L3-L4, L4-L5, L5-L5) }\end{array}$ & $\begin{array}{c}\text { Methicillin-resistant Staphylococcus aureus } \\
\text { (MRSA) }\end{array}$ \\
\hline 2 & Degenerative Disc disease(L4-L5, L5-L5) & Enterobactor \\
\hline 3 & Renal Transplant & Escherichia Coli \\
\hline 4 & Fracture Right Tibia \& fibula shaft & Pseudomonas \\
\hline 5 & Post traumatic nasal deformity & Klebsiella pneumoniae \\
\hline 6 & CABG & Methicillin-sensitive Staphylococcus aureus \\
\hline 7 & Kidney Transplant donor \\
\hline
\end{tabular}

Appropriate decision-making regarding use or non-use of prophylactic antibiotics, choice of antibiotic and duration of prophylactic antibiotic use were 4 parameters with the least adherence to the standard guidelines in the present study. Prophylactic antibiotics were administered in $83 \%$ of the procedures. The timing of administration of prophylactic antibiotics is important and this was correct in $83 \%$ of the surgeries performed. In addition, appropriate antibiotic timing can decrease overall hospitalization costs (Kulkarni and Kochhar, 2005).

According to CDC guidelines, all antibiotic administration must be completed at time of surgical incision, no more than 30 min prior. In our study $83 \%$ percent of patients received antibiotics $30 \mathrm{~min}$ before incision (Figure 4). 


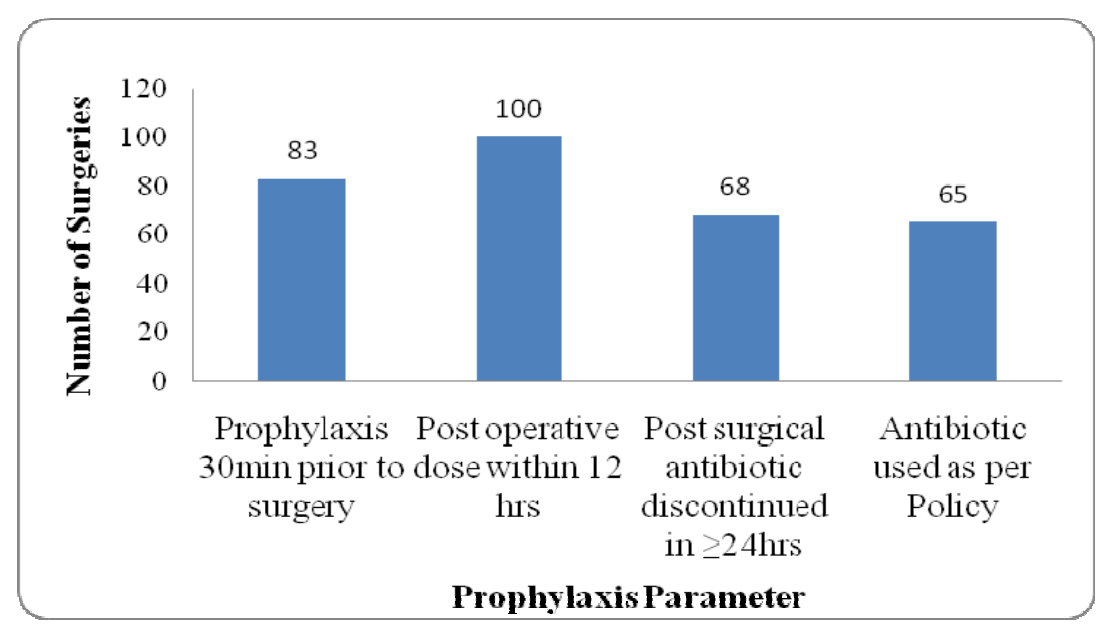

Fig. 4. Summary of antibiotic prophylaxis

As far as duration of antimicrobial prophylaxis is concerned, one antibiotic dose is sufficient for operations lasting $4 \mathrm{~h}$ or less (ASHP, 1999) and further doses may be required in prolonged surgeries because persistence of tissue concentrations past the period of surgery and recovery from anesthesia doesn't improve efficacy but shall increases toxicity and cost of therapy. Even though evidence from literature fails to support prolonged administration of antibiotics prophylaxis in the hospitals, usage beyond $24 \mathrm{~h}$ is common. Longer courses of antibiotics are falsely believed to be a good preventive measure against SSIs (Huskin et al 1998). Since preoperative prophylactic antibiotics take up a large part of prescribed antibiotics prophylaxis in the hospitals, adherence to standard guidelines regarding the duration of antibiotic prophylaxis would keep costs to a minimum which would be desirable especially in a resource limited setting like ours.

In the present study, antibiotics were discontinued in $68 \%$ of the procedures. According to international guidelines, a single dose of antibiotic is enough for most surgical procedures. Prolonged antibiotic prophylaxis is of no benefit (Hollenbeak et al 2002); also potentially harmful to patients due to toxicity, risk of super-infection and the risk of inducing more bacterial resistance (Dellinger et al 1994). As far as duration of antimicrobial prophylaxis is concerned, one antibiotic dose is sufficient for operations lasting $4 \mathrm{~h}$ or less. Further doses may be required in prolonged surgeries. The choice of antibiotic complied with guidelines in only $65 \%$ of the surgical procedures in this study. This low rate is disappointing, particularly as selection of the appropriate antibiotic for prophylaxis was much higher. The high rate of inappropriate choice of prophylactic antibiotic and unnecessary continuation in our study may be due to the lack of medication protocols and treatment guidelines. In this study only 7 cases of surgical site infection were reported. Cephalosporins are recommended for surgical prophylaxis due to their good safety profile, excellent antimicrobial activity broad spectrum and efficacy against common pathogens.

In keeping with these guidelines, our survey revealed that a combination of third generation cephalosporin and an anti-anaerobic agent was the less popular regimen; prescribed by nearly $4 \%$ of the respondents and $7 \%$ surgeries had used combinations of Cephalosporin with aminoglycoside and anti-anaerobe. Routine addition of an amino glycoside to other agents having broad-spectrum gram-negative coverage, such as third generation Cephalosporin's has been shown to provide no additional benefit. We recommend, it is important to select an antibiotic with narrowest antibacterial spectrum to reduce the emergence of resistance and also because broad spectrum antibiotics may be required later if patient develops serious sepsis. Therefore, the use of third generation Cephalosporin's such as Ceftriaxone and Cefotaxime have to be avoided in surgical prophylaxis.

\section{CONCLUSION}

A great amount of concern has been voiced in the past two decades regarding the widespread use of antimicrobials, leading to emergence of multiple drug-resistant organisms. The SSIs reported during the study period shows that most of the surgical procedures which have been done by using Cephalosporin's were protected of SSIs although the guidelines and procedures 
have some other steps such as surgical wound care, material sterilization etc. The high use of antimicrobials especially for a prolonged duration post operatively is a matter of concern. There is an urgent need to promote rational antimicrobial prescribing among surgeons. The need of the hour is developing and implementing consensus-based national guidelines for treatment of surgical infections and prophylaxis by a multidisciplinary group of experts.

The antimicrobial regimens for intra-abdominal infections should cover common aerobic and anaerobic enteric flora. Single agents like Cefazolin or Ceforoxime are effective. Third generation cephalosporin was widely used in many procedure but it is avoided in all type guidelines. Combination regimens include first, second / third generation cephalosporin plus an anti-anaerobe (preferably Metronidazole), amino glycoside plus an anti-anaerobic, Quinolones plus Metronidazole etc. but, no regimen has been found to be superior to the other. Perhaps the biggest controversy in our study was the uses of antimicrobials in clean

\section{REFERENCES}

Akalin HE. Surgical prophylaxis: the evaluation of guideline in era of cost containment. J. Hosp. Infect. 2002;50(A): S3-7. [DOI: 10.1053/jhin.2001.1121]

ASHP therapeutic guidelines on antimicrobial prophylaxis in surgery. Am. J. Health Syst. Pharm. 1999;56(18): 1839-88. [DOI: 10.7860/JCDR/2016/21587.8327]

Berard F, Gandon J. Postoperative wound infections: the influence of ultraviolet irradiation of the operative room and of various other factors. Ann. Surg. 1964;160(2):1192. [DOI: 10.5958/0974-360X.2016.00164.5].

Bratzler DW, Houck PM, Richards C, Steele L, Dellinger EP, Fry DE, Wright C, Ma A, Carr K, Red L. Use of Antimicrobial Prophylaxis for major surgery: baseline results from the national surgical infection prevention project. Arch. Surg. 2005;140(2):174-182. [DOI: 10.1001/ archsurg.140.2.174]

Page CP, Bohnen JM, Fletcher JR, McManus AT, Solomkin JS, Wittmann DH. Antimicrobial Prophylaxis for Surgical Wounds: A Guideline for Clinical Care. Arch. Surg. 1993; 128(1):79-88. [DOI: 10.3109/17453674.2011.636671].

De Lalla F. Perioperative antibiotic prophylaxis: a critical review. Surg. Infect. 2006;7(2):S37-S39. [DOI: 10.1089/ sur.2006.7.s2-37].

Dellinger EP, Gross PA, Barrett TL, Krause PJ, Martone WJ, McGowan JE Jr, Sweet RL, Wenzel RP. Quality standard for antimicrobial prophylaxis in surgical procedures. Clin. Infect. Dis. 1994;18(3):422-7. [DOI: 10.2 146/ajhp120568].

Hollenbeak CS, Murphy D, Dunagan WC, Fraser VJ. Nonrandom selection and the attributable cost of surgical-site infections. Infect. Control Hosp. Epidemiol. surgery despite of benefits outweigh the risks, has been questioned here for clean surgeries.

The results highlight the challenges of disseminating evidence based protocols systematically into routine clinical practice. Various measures are needed to improve appropriateness of prescriptions and adherence include development of evidence based guidelines in collaboration with surgeons, increased outcome based research to document benefits of appropriate antibiotic use, continuing education to disseminate information to practitioners, surveys of antibiotic use and reassessment of prescribing practices over time and providing regular feedback and organizing group education.

The present survey have limitations in the point that the day to day prescribing habits of the surgeons may be different from that put down on paper for the survey and rationality of the prescription cannot be assessed without patient details. The less number of reporting of SSIs could not track every visit of post operative patients.

2002;23(4):177-82. [DOI: 10.1086/650755].

Horan TC, Culver DH, Gaynes RP, Jarvis WR, Edwards JR, Reid CR. Nosocomial infections in surgical patients in the United States. Infect. Control Hosp. Epidemiol. 1993; 14(2):73-80. [DOI: 10.1016/S0196-6553(00)90006-3].

Huskin WC, Soule BM, O'Boyle C, Gulácsi L, O'Rourke EJ, Goldmann DA. Hospital infection prevention and control: A model for improving the quality of hospital care in low- and middle-income countries. Infect. Control Hosp. Epidemiol. 1998;19(2):125-35. [DOI: 10.2307/30142003].

Kulkarni RA, Kochhar PH, Dargude VA, Rajadhyakshya SS, Thatte UM. Patterns of antimicrobial use by surgeons in India. Ind. J. Surg. 2005;67(6):308-15.

Manian FA, Meyer L. Comprehensive surveillance of surgical wound infections in outpatient and inpatient surgery. Infect. Control Hosp. Epidemiol. 1990;11(10):515 -20. [DOI: 10.2307/30151318].

McDonald M, Grabsch E, Marshall C, Forbes A. Single versus multiple dose antimicrobial prophylaxis for major surgery: A systematic review. Aust. NZJ Surg. 1998; 68(6):388-96. [DOI: 10.5958/0974-360X.2016.00164.5].

Olson MM, Lee JT Jr. Continuous 10 years wound infection surveillance. Results, advantages, and unanswered question. Arch. Surg.1990;125(6):794-803. [DOI: 10.101 6/S1070-3241(16)30248-6].

Platt R, Zaleznik DF, Hopkins CC, Dellinger EP, Karchmer AW, Bryan CS, Burke JF, Wikler MA, Marino SK, Holbrook KF, Tosteson TD, Segal MR. Perioperative antibiotics prophylaxis for herniorrhaphy and breast surgery. $N$. Engl. J. Med. 1990;322:153-60. [DOI: 10.1056/NEJM1990 01183220303]. 